\title{
Well-posedness results for a class of toxicokinetic models*
}

\author{
H.T. Banks ${ }^{\dagger}$ and Laura K. Potter ${ }^{\ddagger}$ \\ Center for Research in Scientific Computation \\ North Carolina State University \\ Raleigh, NC 27695-8205 USA
}

July 24, 2001

\begin{abstract}
In this paper we address theoretical issues related to a class of nonlinear parabolic systems used in toxicokinetic modeling. We establish the wellposedness of these systems in a weak or variational setting by proving the existence of a unique solution which depends continuously on the data. These results are then applied specifically to the model equations for a physiologically based pharmacokinetic model for the systemic transport of trichloroethylene (TCE), which is the motivating example for our efforts here.

In addition, we present theoretical results for the parameter estimation problem associated with the general class of abstract systems and the example TCE model. These results further establish the convergence of the Galerkin approximations used in the numerical scheme for the TCE model.
\end{abstract}

\section{Introduction}

In this paper we present theoretical results related to a class of physiologically based pharmacokinetic (PBPK) models. PBPK models are widely used in the field of toxicology to describe the uptake, systemic transport and elimination of compounds within humans and animals. These compartmental models lead to a system of

*2000 Mathematics Subject Classification. 35A05, 37N25, 92C99, 47J30

$\dagger$ Author to whom correspondence should be addressed.

$\ddagger$ Current address: National Health and Environmental Effects Research Laboratory, U.S. Environmental Protection Agency, Research Triangle Park, NC 27711. 
coupled algebraic and differential equations, where each equation represents the transport of the compound through a given organ or tissue. The standard PBPK models, known as the perfusion-limited and diffusion-limited models, are ODEbased models that are derived using simple mass balance principles and assumptions of uniformity and rapid equilibrium [20].

In some cases, the assumption of a "well-mixed" compartment is not appropriate for tissues with heterogeneous physiological properties. For example, large degrees of spatial variations in cell size, lipid distribution, and membrane permeability within adipose (fat) tissue may lead to spatially-dependent concentrations of highly lipophilic (fat-soluble) compounds there. To more accurately capture the effects of these tissue heterogeneities, specialized PDE-based compartmental models can be developed by incorporating the specific physiology of the tissue into the model assumptions. The resulting compartment is then coupled with the standard PBPK compartments for the remaining organs and tissues, yielding a hybrid PBPK whole-body model.

Examples of hybrid PBPK models include a CFD-PBPK model for nasal gas and vapor uptake [12], a distributed parameter-PBPK model for inhalation and dermal absorption [26], and a hybrid PBPK model for the systemic transport of trichloroethylene (TCE). The TCE model, which motivates our efforts here, includes a specialized axial dispersion-based model for the transport of TCE in the adipose tissue, where TCE is known to accumulate [29].

TCE is a highly lipophilic solvent that has been used widely in industry as a metal degreasing agent. Now a common soil and groundwater contaminant, TCE can be found at Superfund sites and Department of Defense facilities across the United States [28]. Humans come into contact with TCE most often by inhaling TCE vapor in an industrial setting, or more commonly by drinking contaminated water.

TCE and several of its metabolites are known to produce toxic effects in humans. Acute exposure to TCE impacts the central nervous system, causing symptoms such as fatigue, headaches, dizziness and drowsiness [16]. Several of its metabolites are suspected of causing toxicity in animals and/or humans [10, 21], including liver, lung and kidney tumors [9, 10, 11, 17], as well as developmental defects [8, 27].

A key property of TCE that greatly affects its pharmacokinetics is its high degree of lipophilicity, which leads to the accumulation of TCE in the adipose tissue. Numerous studies have demonstrated that adipose tissue is highly heterogeneous, with wide variations in fat cell size, metabolic activity, blood flow rates, and membrane permeability $[13,14,18,25]$. Moreover, the amount of lipid in each adipocyte is 
known to vary, creating an uneven distribution of lipids across the tissue [18]. Since TCE and other compounds of interest are highly lipophilic, this uneven distribution of lipids may lead to an uneven distribution of the compounds themselves. This would then result in a spatially dependent concentration function for such a compound inside the adipose tissue.

In Section 2 we present an overview of the TCE PBPK-hybrid model, including the state space settings and weak formulation. This model is the motivation for the theoretical results we develop here for a general abstract class of nonlinear parabolic systems that may arise in toxicokinetic modeling. In Section 3 we establish the existence of unique weak solutions for the general class of problems and the TCE PBPK-hybrid model. Finally, in Section 4 we address theoretical issues related to the associated parameter estimation problem. The results presented in this paper also provide a framework for a numerical scheme that we implement computationally in [22] for model simulation and parameter identification. Moreover, we establish the theoretical convergence of numerical solutions to the solution for the infinitedimensional system of model equations.

\section{The TCE PBPK-hybrid model}

In this section we provide an overview of the TCE PBPK-hybrid model, which is developed and discussed in full detail in [2, 22]. The compartmental model for the adipose tissue is based on the axial dispersion model of Roberts and Rowland [24] for the transport of solutes in the liver, and is adapted for the specific physiology of adipose tissue. This aggregate model uses a representative "cell" to capture the transport behavior in a collection of many similar "cells" that have varying properties.

In this particular case, the representative unit includes a single adipocyte (fat cell) with an adjoining capillary, both immersed in the surrounding interstitial fluid. As discussed in [2, 22], the adipocyte is represented by a sphere centered at the origin with radius $r_{1}$, and the capillary is a cylindrical tube with circular cross-section and central axis $r=r_{2}+\alpha, \theta=\theta_{0}, \varepsilon_{1}<\phi<\varepsilon_{2}$ in spherical coordinates. The interstitial fluid fills in the space surrounding the other two regions.

We assume that blood and TCE enter the representative adipose capillary region from the arterial blood system. The transport within the capillary is described by a one-dimensional convection-dispersion equation, while the exchange between the capillary and the other two adipose regions (adipocyte and interstitial space) is modeled using Fick's first law of diffusion. Moreover, we use flux balance to derive 
boundary conditions that connect the capillary region to the arterial and venous blood systems. Within each of the adipocyte and interstitial regions, we have a two-dimensional diffusion equation, as well as the terms representing the passive transport between all three regions. The boundary conditions in the adipocyte and interstitial space are standard periodic and finiteness conditions that are appropriate for diffusion on a spherical domain.

The other tissue compartments used in the PBPK-hybrid model correspond to the brain, kidney, liver, muscle and remaining tissues, and each is modeled as a perfusion-limited compartment. We assume uptake via inhalation in the

lungs, which is modeled using a steady-state assumption. Moreover, we include a Michaelis-Menten term in the liver to represent the metabolism of TCE there. See $[2,22]$ for a detailed derivation and discussion of the model equations.

\subsection{State space setting and weak formulation}

Here we present the weak or variational formulation for the TCE PBPK-hybrid model. First we consider the three regions of the adipose tissue compartment. The domain for the capillary region of the adipose tissue is given by the arc

$$
\bar{\Omega}_{B}=\left\{(r, \theta, \phi): r=r_{1}, \theta=\theta_{0}, \varepsilon_{1}<\phi<\pi-\varepsilon_{2}\right\}
$$

in spherical coordinates, and the domain for the adipocyte and interstitial regions is the spherical shell

$$
\bar{\Omega}_{I A}=\left\{(r, \theta, \phi): r=r_{1}, 0 \leq \theta \leq 2 \pi, 0 \leq \phi \leq \pi\right\} .
$$

Note that both $r$ and $\theta$ are constant in the domain $\bar{\Omega}_{B}$, so that we may write $u=u(\phi)$ for functions $u \in \bar{\Omega}_{B}$. Similarly, since $r$ is constant in $\bar{\Omega}_{I A}$ we write $u=u(\theta, \phi)$ for $u \in \bar{\Omega}_{I A}$. In both cases, $r=r_{1}$ is understood.

We choose the following state spaces for the regions of the adipose dispersion model: $H_{B}^{1}=H^{1}\left(\bar{\Omega}_{B}\right)$ in the capillary region and

$$
H_{I A}^{1} \equiv H_{p e r}^{1}\left(\bar{\Omega}_{I A}\right)=\left\{u \in H^{1}\left(\bar{\Omega}_{I A}\right): u(\theta, \phi)=u(\theta+2 \pi, \phi)\right\}
$$

in the interstitial and adipocyte regions. The norms and inner products on these 
spaces are defined by

$$
\begin{array}{rlrl}
\langle u, v\rangle_{B} & =\int_{\varepsilon_{1}}^{\pi-\varepsilon_{2}} u(\phi) v(\phi) \sin \phi d \phi & & \text { for } u, v \in L^{2}\left(\bar{\Omega}_{B}\right), \\
|u|_{B}^{2} & =\int_{\varepsilon_{1}}^{\pi-\varepsilon_{2}}|u(\phi)|^{2} \sin \phi d \phi & & \text { for } u \in L^{2}\left(\bar{\Omega}_{B}\right), \\
|u|_{H_{B}^{1}}^{2} & =\int_{\varepsilon_{1}}^{\pi-\varepsilon_{2}}\left(\left|\frac{1}{r_{1}} \frac{\partial u(\phi)}{\partial \phi}\right|^{2}+|u(\phi)|^{2}\right) \sin \phi d \phi & & \text { for } u \in H^{1}\left(\bar{\Omega}_{B}\right), \\
\langle u, v\rangle_{I A} & =\int_{0}^{2 \pi} \int_{0}^{\pi} u(\theta, \phi) v(\theta, \phi) \sin \phi d \phi d \theta & & \text { for } u, v \in L^{2}\left(\bar{\Omega}_{I A}\right), \\
|u|_{I A}^{2} & =\int_{0}^{2 \pi} \int_{0}^{\pi}|u(\theta, \phi)|^{2} \sin \phi d \phi d \theta & & \text { for } u \in L^{2}\left(\bar{\Omega}_{I A}\right), \\
|u|_{H_{I A}^{1}}^{2} & =\int_{0}^{2 \pi} \int_{0}^{\pi}\left(\left|\frac{1}{r_{1} \sin \phi} \frac{\partial u(\theta, \phi)}{\partial \theta}\right|^{2}\right. & \\
& \left.+\left|\frac{1}{r_{1}} \frac{\partial u(\theta, \phi)}{\partial \phi}\right|^{2}+|u(\theta, \phi)|^{2}\right) \sin \phi d \phi d \theta & & \text { for } u \in H_{p e r}^{1}\left(\bar{\Omega}_{I A}\right) .
\end{array}
$$

Note that in spherical coordinates, for $u \in H^{1}\left(\bar{\Omega}_{B}\right)$ we have

$$
\nabla u=\left(\frac{\partial u}{\partial r}, \frac{1}{r \sin \phi} \frac{\partial u}{\partial \theta}, \frac{1}{r} \frac{\partial u}{\partial \phi}\right)=\left(0,0, \frac{1}{r_{1}} \frac{\partial u}{\partial \phi}\right),
$$

and for $u \in H^{1}\left(\bar{\Omega}_{I A}\right)$ we have

$$
\nabla u=\left(\frac{\partial u}{\partial r}, \frac{1}{r \sin \phi} \frac{\partial u}{\partial \theta}, \frac{1}{r} \frac{\partial u}{\partial \phi}\right)=\left(0, \frac{1}{r_{1} \sin \phi} \frac{\partial u}{\partial \theta}, \frac{1}{r_{1}} \frac{\partial u}{\partial \phi}\right) .
$$

We now define the state space $\mathcal{V}=H_{B}^{1} \times H_{I A}^{1} \times H_{I A}^{1} \times \mathbb{R}^{6}$ and the space $\mathcal{H}=L^{2}\left(\bar{\Omega}_{B}\right) \times L^{2}\left(\bar{\Omega}_{I A}\right) \times L^{2}\left(\bar{\Omega}_{I A}\right) \times \mathbb{R}^{6}$, with corresponding norms and inner product

$$
\begin{array}{rlr}
|u|_{\mathcal{V}}^{2}=\left|u_{1}\right|_{H_{B}^{1}}^{2}+\left|u_{2}\right|_{H_{I A}^{1}}^{2}+\left|u_{3}\right|_{H_{I A}^{1}}^{2}+\sum_{i=4}^{9}\left|u_{i}\right|^{2} & \text { for } u \in \mathcal{V}, \\
|u|_{\mathcal{H}}^{2}=\left|u_{1}\right|_{B}^{2}+\left|u_{2}\right|_{I A}^{2}+\left|u_{3}\right|_{I A}^{2}+\sum_{i=4}^{9}\left|u_{i}\right|^{2} & \text { for } u \in \mathcal{H} \\
\langle u, v\rangle=\left\langle u_{1}, v_{1}\right\rangle_{B}+\left\langle u_{2}, v_{2}\right\rangle_{I A}+\left\langle u_{3}, v_{3}\right\rangle_{I A}+\sum_{i=4}^{9} u_{i} v_{i} & \text { for } u, v \in \mathcal{H} .
\end{array}
$$

Let $\Gamma$ denote the boundary of the domain $\bar{\Omega}_{I A}$. Since $\bar{\Omega}_{I A}$ is defined as a spherical shell in spherical coordinates, we can choose the boundary $\Gamma$ to be any fixed value of $\theta$. Therefore we define $\Gamma=\left\{(r, \theta, \phi): r=r_{1}, \theta=\theta_{0}, 0 \leq \phi \leq \pi\right\}$. Moreover, we define the trace operator $T_{\theta_{0}}^{0}: H^{1}\left(\bar{\Omega}_{I A}\right) \rightarrow L^{2}(\Gamma)$ so that

$$
T_{\theta_{0}}^{0} u=\left.u\right|_{\theta=\theta_{0}}
$$

for any $u \in H^{1}\left(\bar{\Omega}_{I A}\right)$, where

$$
|u|_{L^{2}(\Gamma)}^{2}=\int_{0}^{\pi}|u(\phi)|^{2} \sin \phi d \phi
$$


for all $u \in L^{2}(\Gamma)$, and where $|u|_{H^{1}\left(\bar{\Omega}_{I A}\right)}=|u|_{H_{I A}^{1}}$.

It follows from the Trace Theorem (see Theorem 6 in [23], p. 240) that there exists $K_{1}>0$ such that for every $u \in H^{1}\left(\bar{\Omega}_{I A}\right)$ we have

$$
\left|T_{\theta_{0}}^{0} u\right|_{L^{2}(\Gamma)} \leq K_{1}|u|_{H_{I A}^{1}}
$$

Here we are seeking a solution $y(t) \in \mathcal{V}$ which satisfies the initial condition $y(0)=y_{0} \in \mathcal{H}$ and

$$
\langle\dot{y}(t), \psi\rangle_{\mathcal{V}^{*}, \mathcal{V}}+\sigma(y(t), \psi)+\langle g(y(t)), \psi\rangle_{\mathcal{H}}=\langle f(t), \psi\rangle_{\mathcal{V}^{*}, \mathcal{V}}
$$

for all $\psi \in \mathcal{V}$, where

$$
\begin{aligned}
& \sigma(u, v)=\left\langle\frac{a_{1}}{r_{1}^{2}} \frac{\partial u_{1}}{\partial \phi}-\frac{a_{2}}{r_{1}} u_{1}, \frac{\partial v_{1}}{\partial \phi}\right\rangle_{B}+\left\langle a_{3} u_{1}-a_{4} T_{\theta_{0}}^{0} u_{2}, v_{1}\right\rangle_{B} \\
& +\left\langle a_{5} u_{1}-a_{6} T_{\theta_{0}}^{0} u_{3}, v_{1}\right\rangle_{B}+\left\langle-a_{7} u_{4}, v_{1}\left(\varepsilon_{1}\right)\right\rangle_{\mathbb{R}}+\left\langle a_{8} u_{4}, v_{1}\left(\pi-\varepsilon_{2}\right)\right\rangle_{\mathbb{R}} \\
& +\left\langle\frac{b_{1}}{r_{1}^{2} \sin ^{2} \phi} \frac{\partial u_{2}}{\partial \theta}, \frac{\partial v_{2}}{\partial \theta}\right\rangle_{I A}+\left\langle\frac{b_{1}}{r_{1}^{2}} \frac{\partial u_{2}}{\partial \phi}, \frac{\partial v_{2}}{\partial \phi}\right\rangle_{I A} \\
& +\left\langle\delta_{\theta_{0}}(\theta) \chi_{B}(\phi)\left(-b_{2} u_{1}+b_{3} u_{2}\right)+b_{4} u_{2}-b_{5} u_{3}, v_{2}\right\rangle_{I A} \\
& +\left\langle\frac{c_{1}}{r_{1}^{2} \sin ^{2} \phi} \frac{\partial u_{3}}{\partial \theta}, \frac{\partial v_{3}}{\partial \theta}\right\rangle_{I A}+\left\langle\frac{c_{1}}{r_{1}^{2}} \frac{\partial u_{3}}{\partial \phi}, \frac{\partial v_{3}}{\partial \phi}\right\rangle_{I A} \\
& +\left\langle\delta_{\theta_{0}}(\theta) \chi_{B}(\phi)\left(-c_{2} u_{1}+c_{3} u_{3}\right)+c_{4} u_{3}-c_{5} u_{2}, v_{3}\right\rangle_{I A} \\
& +\left\langle-d_{1} u_{1}\left(\pi-\varepsilon_{2}\right)+d_{2} u_{4}-d_{3} u_{5}-d_{4} u_{6}-d_{5} u_{7}-d_{6} u_{8}-d_{7} u_{9}, v_{4}\right\rangle_{\mathbb{R}} \\
& +\left\langle-e_{1} u_{4}+e_{2} u_{5}, v_{5}\right\rangle_{\mathbb{R}}+\left\langle-h_{1} u_{4}+h_{2} u_{6}, v_{6}\right\rangle_{\mathbb{R}}+\left\langle-\ell_{1} u_{4}+\ell_{2} u_{7}, v_{7}\right\rangle_{\mathbb{R}} \\
& +\left\langle-p_{1} u_{4}+p_{2} u_{8}, v_{8}\right\rangle_{\mathbb{R}}+\left\langle-s_{1} u_{4}+s_{2} u_{9}, v_{9}\right\rangle_{\mathbb{R}} \\
& g(y)=\left[0,0,0,0,0,0,0,0, g_{9}\left(y_{9}\right)\right]^{\mathrm{T}} \\
& f(t)=\left[\begin{array}{c}
\frac{V_{B} Q_{p} Q_{c}}{1000 A_{B} r_{1}\left(Q_{c}+\frac{Q_{p}}{P_{b}}\right)} C_{c}(t) \delta_{\varepsilon_{1}}(\phi) \\
0 \\
0 \\
0 \\
\frac{Q_{b r} Q_{c}}{Q_{c}+\frac{Q_{p}}{P_{b}}} C_{c}(t) \\
\frac{Q_{k} Q_{c}}{Q_{c}+\frac{Q_{p}}{P_{b}}} C_{c}(t) \\
\frac{Q_{m} Q_{c}}{Q_{c}+\frac{Q_{p}}{P_{b}}} C_{c}(t) \\
\frac{Q_{t} Q_{c}}{Q_{c}+\frac{Q_{p}}{P_{b}}} C_{c}(t) \\
\frac{Q_{l} Q_{c}}{Q_{c}+\frac{Q_{p}}{P_{b}}} C_{c}(t)
\end{array}\right],
\end{aligned}
$$

with

$$
g_{9}\left(y_{9}\right)=\left\{\begin{array}{cc}
\frac{s_{3} y_{9}}{1+s_{4} y_{9}} & \text { if } y_{9} \geq 0 \\
0 & \text { otherwise. }
\end{array}\right.
$$


The vector $y$ represents TCE concentrations (in $\mathrm{mg} / \mathrm{liter}$ ) in each of the tissue compartments and subcompartments. Specifically, we have

$$
y(t)=\left[C_{B}(t), C_{I}(t), C_{A}(t), C_{v}(t), C_{b r}(t), C_{k}(t), C_{m}(t), C_{t}(t), C_{l}(t)\right]^{\mathrm{T}}
$$

where $C_{B}(t), C_{I}(t)$ and $C_{A}(t)$ represent TCE concentrations in the capillary, interstitial and adipocyte regions of the adipose tissue respectively, and $C_{v}(t), C_{b r}(t)$, $C_{k}(t), C_{m}(t), C_{l}(t)$ and $C_{t}(t)$ denote TCE concentrations in the venous blood, brain, kidney, muscle, liver and remaining tissue compartments, respectively. The function $C_{c}(t)$ that appears in the forcing function (5) is the concentration of TCE in the chamber air, and is a specified quantity. See [2, 22] for details.

Here we use the notation $\dot{y}=\frac{\partial y}{\partial t}$, and $\langle\cdot, \cdot\rangle_{\mathcal{V}^{*}, \mathcal{V}}$ denotes the usual duality product described in the next section. The system (2) belongs to a general class of abstract problems that we consider in the next section, where we address issues of wellposedness.

\section{Well-posedness of solutions}

First we present well-posedness results for a general class of abstract nonlinear parabolic equations. The theoretical issues that we address here establish the existence, uniqueness and continuous dependence of solutions for the TCE PBPKhybrid model, as well as for a class of more general equations that may arise in more complicated models of xenobiotic transport inside the body. Moreover, these theoretical efforts lay the groundwork for computational methods used in model verification, simulation and parameter estimation.

The main result that we establish here regarding well-posedness of solutions is based on ideas presented in [5] and [1]. Banks and Musante [5] proved well-posedness results for two classes of abstract nonlinear parabolic systems. The first class they addressed requires the nonlinearity to satisfy a particular convexity condition. The second class replaces the convexity condition with an assumption that the forcing function has additional regularity with respect to time or space.

The TCE PBPK-hybrid model has a simple Michaelis-Menten nonlinearity in the liver equation, and it can be shown that this system of model equations fits into the first of these abstract classes of problems. Although this happens to be the case for TCE, which has a simple metabolic mechanism, there may be other lipophilic compounds which have more complicated kinetics (e.g., protein binding) that would not satisfy the convexity condition.

The forcing function in our TCE model is related to the inhalation of TCE va- 
por in the air, and does not have the necessary regularity required for the model to fit into the second class of abstract problems discussed by Banks and Musante. Transport models for other inhaled compounds would have a similar forcing function that also may lack the required regularity. Therefore, a model for an inhaled lipophilic compound with complicated nonlinear kinetics would likely fall outside both of these abstract classes of problems.

Using ideas in [1], we can improve upon the results of Banks and Musante by achieving well-posedness for a more general class of abstract nonlinear parabolic equations. Ackleh, Banks and Pintér [1] proved the well-posedness of second order elastomer problems in which the nonlinearities satisfy local Lipschitz and affine domination properties. Our result is based on their work, and will establish wellposedness for a wider class of inhaled xenobiotic transport models.

\subsection{Well-posedness results for a class of abstract nonlinear parabolic systems}

Consider the system

$$
\begin{aligned}
& \dot{y}(t)+\mathcal{A} y(t)+g(y(t))=f(t) \quad \text { in } \mathcal{V}^{*} \\
& y(0)=y_{0}
\end{aligned}
$$

for $t \in(0, T)$ with $T<\infty$. We assume that $\mathcal{V}, \mathcal{H}$ and $\mathcal{V}^{*}$ are separable real Hilbert spaces that form a Gelfand triple [30] which satisfies

$$
\mathcal{V} \hookrightarrow \mathcal{H} \simeq \mathcal{H}^{*} \hookrightarrow \mathcal{V}^{*} .
$$

We denote the inner product in $\mathcal{H}$ by $\langle\cdot, \cdot\rangle$, and the norms in each of the spaces $\mathcal{V}$, $\mathcal{H}$ and $\mathcal{V}^{*}$ are denoted by $|\cdot|_{\mathcal{V}},|\cdot|$ and $|\cdot|_{\mathcal{V}}$ respectively. Moreover, we assume that the embedding $\mathcal{V} \hookrightarrow \mathcal{H}$ is dense and continuous, with

$$
|\psi| \leq k|\psi|_{\mathcal{V}} \quad \text { for all } \quad \psi \in \mathcal{V} .
$$

The duality product $\langle\cdot, \cdot\rangle_{\mathcal{V}^{*}, \mathcal{V}}$ is the extension by continuity of the inner product in $\mathcal{H}$ from $\mathcal{H} \times \mathcal{V}$ to $\mathcal{V}^{*} \times \mathcal{V}$ (see [30] for a complete discussion).

We assume the operator $\mathcal{A}$ is defined in terms of a given sesquilinear form $\sigma$ : $\mathcal{V} \times \mathcal{V} \rightarrow \mathbb{R}$. That is, we define $\mathcal{A}: \mathcal{V} \rightarrow \mathcal{V}^{*}$ by $\langle\mathcal{A} u, v\rangle_{\mathcal{V}^{*}, \mathcal{V}}=\sigma(u, v)$, and it follows under the assumptions on $\sigma$ below that $\mathcal{A} \in \mathcal{L}\left(\mathcal{V}, \mathcal{V}^{*}\right)$.

We make the following standing assumptions on $\sigma, f$ and $g$ :

(A1) The sesquilinear form $\sigma$ is bounded in $\mathcal{V}$, i.e., there exists $C_{1}>0$ such that

$$
|\sigma(u, v)| \leq C_{1}|u|_{\mathcal{V}}|v|_{\mathcal{V}} \quad \text { for all } u, v \in \mathcal{V} .
$$


(A2) The sesquilinear form $\sigma$ is elliptic on $\mathcal{V}$. That is, there exist $k_{1}>0$ and $\lambda>0$ such that

$$
\sigma(u, u) \geq k_{1}|u|_{\mathcal{V}}^{2}-\lambda|u|^{2} \quad \text { for all } u \in \mathcal{V}
$$

(A3) The forcing function $f$ satisfies

$$
f \in L_{2}\left((0, T) ; \mathcal{V}^{*}\right)
$$

(A4) The nonlinear function $g: \mathcal{H} \rightarrow \mathcal{H}$ satisfies the following local Lipschitz condition: let $B_{r}(0)=\{u \in \mathcal{H}:|u| \leq r\}$ denote the ball of radius $r$ centered around the origin in $\mathcal{H}$. Then given $r>0$, there exists $L_{B_{r}}>0$ such that

$$
|g(u)-g(v)| \leq L_{B_{r}}|u-v| \quad \text { for all } u, v \in B_{r}(0) .
$$

(A5) There exist positive constants $C_{2}$ and $C_{3}$ such that

$$
|g(u)| \leq C_{2}|u|+C_{3} \quad \text { for all } u \in \mathcal{H}
$$

We say that $y \in L_{2}((0, T) ; \mathcal{V})$ is a solution of $(7),(8)$ if it satisfies

$$
\begin{aligned}
& \langle\dot{y}(t), \psi\rangle_{\mathcal{V}^{*}, \mathcal{V}}+\sigma(y(t), \psi)+\langle g(y(t)), \psi\rangle=\langle f(t), \psi\rangle_{\mathcal{V}^{*}, \mathcal{V}} \\
& y(0)=y_{0}
\end{aligned}
$$

for all $\psi \in \mathcal{V}$.

Theorem 3.1 (Local existence) Under Assumptions (A1) - (A4) and for any $y_{0} \in$ $\mathcal{H}$, the system $(15)$ - (16) has a unique solution $y \in L_{2}((0, T) ; \mathcal{V}) \cap C([0, T] ; \mathcal{H})$ on some interval $\left[0, t^{*}\right]$.

Proof: Let $\mathcal{P}$ denote the Hilbert space radial retraction onto the ball in $\mathcal{H}$ with radius 1 centered at $y_{0}$, and define the nonlinear function $\tilde{g}: \mathcal{H} \rightarrow \mathcal{H}$ by

$$
\tilde{g}(u)=g(\mathcal{P} u) .
$$

Then

$$
|\tilde{g}(u)-\tilde{g}(v)| \leq L_{B_{\left(\left|y_{0}\right|+1\right)}}|\mathcal{P} u-\mathcal{P} v| \leq \tilde{L}|u-v|
$$

for all $u, v \in \mathcal{H}$, where $\tilde{L}=2$ (see Lemma 2.1, p. 230 in [4]). Moreover,

$$
|\tilde{g}(u)| \leq \tilde{L}|u|+c
$$

where $c=|g(0)| \geq 0$ since

$$
|\tilde{g}(u)|-|\tilde{g}(0)| \leq|\tilde{g}(u)-\tilde{g}(0)| \leq \tilde{L}|u| .
$$


Now consider the problem

$$
\begin{aligned}
& \dot{y}(t)+\mathcal{A} y(t)+\tilde{g}(y(t))=f(t) \quad \text { in } \mathcal{V}^{*} \\
& y(0)=y_{0},
\end{aligned}
$$

which is equivalent to

$$
\begin{aligned}
& \langle\dot{y}(t), \psi\rangle_{\mathcal{V}^{*}, \mathcal{V}}+\sigma(y(t), \psi)+\langle\tilde{g}(y(t)), \psi\rangle=\langle f(t), \psi\rangle_{\mathcal{V}^{*}, \mathcal{V}} \\
& y(0)=y_{0}
\end{aligned}
$$

for all $\psi \in \mathcal{V}$.

Suppose that there exists a solution $y(t)$ to $(21)-(22)$ on an interval $[0, T]$. Here we establish a priori bounds for this solution by setting $\psi=y(t)$ in $(21)$ and integrating from 0 to $t$ for some $t \in[0, T]$ :

$$
\frac{1}{2}|y(t)|^{2}+\int_{0}^{t}[\sigma(y(s), y(s))+\langle\tilde{g}(y(s)), y(s)\rangle] d s=\frac{1}{2}|y(0)|^{2}+\int_{0}^{t}\langle f(s), y(s)\rangle_{\mathcal{V}^{*}, \mathcal{V}} d s .
$$

Moreover, using (11), (18), (22) and standard inequalities we obtain

$$
\begin{aligned}
|y(t)|^{2}+k_{1} \int_{0}^{t}|y(s)|_{\mathcal{V}}^{2} d s & \leq\left|y_{0}\right|^{2}+\frac{1}{k_{1}} \int_{0}^{T}|f(s)|_{\mathcal{V}^{*}}^{2} d s+c^{2} T+\hat{L} \tilde{C} T \\
& \equiv \hat{C}\left(k_{1}, f, y_{0}, c, T, \hat{L}, \tilde{C}\right) .
\end{aligned}
$$

This establishes an a priori bound for any solution of the system (21) - (22).

Next we define the "Galerkin" approximations for (19) by

$$
y^{N}(t)=\sum_{k=1}^{N} c_{k}^{N}(t) \psi_{k},
$$

where $\left\{\psi_{k}\right\}_{k=1}^{\infty} \subset \mathcal{V}$ is a linearly independent total subset of $\mathcal{V}$, and where $\left\{c_{k}^{N}(t)\right\}_{k=1}^{N}$ are chosen so that $y^{N}(t)$ is the unique solution of

$$
\left\langle\dot{y}^{N}(t), \psi_{j}\right\rangle+\sigma\left(y^{N}(t), \psi_{j}\right)+\left\langle\tilde{g}\left(y^{N}(t)\right), \psi_{j}\right\rangle=\left\langle f(t), \psi_{j}\right\rangle_{\mathcal{V}^{*}, \mathcal{V}}
$$

for $j=1, \ldots, N$ with $c_{k}^{N}(0)=c_{0 k}^{N}$. Since $\left\{\psi_{k}\right\}_{k=1}^{\infty}$ is a total subset of $\mathcal{V}$, we can and do choose the constants $\left\{c_{0 k}^{N}\right\}$ so that

$$
y_{0}=\lim _{N \rightarrow \infty} \sum_{k=1}^{N} y_{0}^{N} \equiv \lim _{N \rightarrow \infty} \sum_{k=1}^{N} c_{0 k}^{N} \psi_{k} \quad \text { in } \mathcal{V} .
$$

Now we multiply (24) by $c_{j}^{N}(t)$ and sum over $j=1, \ldots, N$ :

$$
\frac{1}{2} \frac{d}{d t}\left[\left|y^{N}(t)\right|^{2}\right]+\sigma\left(y^{N}(t), y^{N}(t)\right)+\left\langle\tilde{g}\left(y^{N}(t)\right), y^{N}(t)\right\rangle=\left\langle f(t), y^{N}(t)\right\rangle_{\mathcal{V}^{*}, \mathcal{V}}
$$


Note that $\left|y_{0}^{N}\right|_{\mathcal{V}}$ is uniformly bounded by some constant $K_{0}$ since $y_{0}^{N}$ converges strongly to $y_{0}$ as in (25), and therefore we can use arguments similar to those above to obtain the uniform bound

$$
\left|y^{N}(t)\right|^{2}+k_{1} \int_{0}^{t}\left|y^{N}(s)\right|_{\mathcal{V}}^{2} d s \leq \hat{c}\left(k_{1}, f, K_{0}, c, T, \hat{L}, \tilde{C}\right)
$$

for some $\hat{c}$ which is independent of $N$.

Next we turn to the convergence of the Galerkin approximations. Note that (26) implies that $\left\{y^{N}\right\}$ is bounded in $L^{2}((0, T) ; \mathcal{V})$. Therefore there exists

$y \in L^{2}((0, T) ; \mathcal{V})$ and a subsequence of $\left\{y^{N}\right\}$ (which we will denote by $\left\{y^{N}\right\}$ ) such that

$$
y^{N} \stackrel{w}{\longrightarrow} y \quad \text { in } L^{2}((0, T) ; \mathcal{V}) .
$$

It follows that $y^{N} \stackrel{w}{\longrightarrow} y$ in $L_{2}((0, T) ; \mathcal{H})$, which further implies

$$
y^{N}(t) \stackrel{w}{\longrightarrow} y(t) \quad \text { in } \mathcal{H} \text { for almost every } t \in[0, T] .
$$

Moreover, (18) and (26) imply that $\left\{\tilde{g}\left(y^{N}\right)\right\}$ is bounded in $L_{2}((0, T) ; \mathcal{H})$, so that there exists

$h \in L_{2}((0, T) ; \mathcal{H})$ such that

$$
\tilde{g}\left(y^{N}\right) \stackrel{w}{\longrightarrow} h \quad \text { in } L_{2}((0, T) ; \mathcal{H})
$$

Next we establish that $y$ is a solution to (21), (22). Let $P_{M}$ be the class of functions $\eta \in L_{2}((0, T) ; \mathcal{V})$ that can be represented in the form

$$
\eta(t)=\sum_{k=1}^{M} a_{k}(t) \psi_{k},
$$

where $a_{k} \in C^{1}[0, T]$. Moreover, we define $P=\bigcup_{M=1}^{\infty} P_{M}$, which implies that $P$ is dense in $L_{2}((0, T) ; \mathcal{V})$.

We multiply $(24)$ by $a_{j}(t)$, then sum from 1 to $M$ and integrate over $[0, t]$, integrating by parts in the first term. Then for fixed $\eta \in P_{M}$ for $M \leq N$, we take the limit as $N \rightarrow \infty$ to obtain

$$
\begin{aligned}
\int_{0}^{t}[-\langle y(s), \dot{\eta}(s)\rangle+\sigma(y(s), \eta(s)) & +\langle h(s), \eta(s)\rangle] d s+\langle y(t), \eta(t)\rangle \\
& =\left\langle y_{0}, \eta(0)\right\rangle+\int_{0}^{t}\langle f(s), \eta(s)\rangle_{\mathcal{V}^{*}, \mathcal{V}} d s
\end{aligned}
$$

for almost every $t \in[0, T]$, where we have used the convergences (27), (28) and (29). 
Note that the $M$ we chose above was arbitrary, and since $\left\{\psi_{j}\right\}$ is total in $\mathcal{V}$ it follows that

$$
\int_{0}^{T} \dot{a}(s)\langle-y(s), \psi\rangle d s+\int_{0}^{T} a(s)\left[\sigma(y(s), \psi)+\langle h(s), \psi\rangle-\langle f(s), \psi\rangle_{\mathcal{V}^{*}, \mathcal{V}}\right] d s=0
$$

for every $\psi \in \mathcal{V}$. This equation holds for every $a \in C_{0}^{\infty}[0, T]$, which further implies that $\dot{y} \in L_{2}((0, T) ; \mathcal{V})^{*} \simeq L_{2}\left((0, T) ; \mathcal{V}^{*}\right)$ and

$$
\langle\dot{y}(t), \psi\rangle_{\mathcal{V}^{*}, \mathcal{V}}+\sigma(y(t), \psi)+\langle h(t), \psi\rangle=\langle f(t), \psi\rangle_{\mathcal{V}^{*}, \mathcal{V}}
$$

for each $\psi \in \mathcal{V}$ and for almost every $t \in[0, T]$. Moreover, since $y \in L_{2}((0, T) ; \mathcal{V})$ and $\dot{y} \in L_{2}\left((0, T) ; \mathcal{V}^{*}\right)$, it follows from Theorem 3.1 in $[19$, p. 19] that $y \in C([0, T] ; \mathcal{H})$.

To show that $y(0)=y_{0}$, consider (30) with $\eta=\eta_{j}=a(t) \psi_{j}, a \in C^{1}[0, T]$ and $a(0) \neq 0$, integrating by parts in the first term:

$$
\begin{aligned}
\int_{0}^{t}\left[\langle\dot{y}(s), \eta(s)\rangle_{\mathcal{V}^{*}, \mathcal{V}}\right. & +\sigma(y(s), \eta(s))+\langle h(s), \eta(s)\rangle] d s+\langle y(t), \eta(t)\rangle \\
& +\left.\langle-y(s), \eta(s)\rangle\right|_{s=0} ^{s=t}=\left\langle y_{0}, \eta(0)\right\rangle+\int_{0}^{t}\langle f(s), \eta(s)\rangle_{\mathcal{V}^{*}, \mathcal{V}} d s .(32)
\end{aligned}
$$

It follows from (31) and (32) that $y(0)=y_{0}$.

Finally, we show that $h=\tilde{g}(y)$. If we let $z^{N}(t)=y^{N}(t)-y(t) \in L_{2}((0, T) ; \mathcal{V}) \cap$ $C([0, T] ; \mathcal{H})$, and combine $(24)$ with $\psi_{j}=y^{N}(t)$ and $(31)$ with $\psi=y(t)$, integrating from 0 to $t$ and adding, we obtain

$$
\begin{aligned}
\left|z^{N}(t)\right|^{2} & +2 \int_{0}^{t} \sigma\left(z^{N}(s), z^{N}(s)\right) d s=\left|y^{N}(0)-y_{0}\right|^{2}+2 \int_{0}^{t}\left\langle f(s), z^{N}(s)\right\rangle_{\mathcal{V}^{*}, \mathcal{V}} d s \\
& +4 \int_{0}^{t}\langle f(s), y(s)\rangle_{\mathcal{V}^{*}, \mathcal{V}} d s-2 \int_{0}^{t}\left\langle\tilde{g}\left(y^{N}(s)\right)-h(s), z^{N}(s)\right\rangle d s \\
& -2 \int_{0}^{t}\left\langle h(s), y^{N}(s)\right\rangle d s-2 \int_{0}^{t}\left\langle\tilde{g}\left(y^{N}(s)\right), y(s)\right\rangle d s-2\left\langle y(t), y^{N}(t)\right\rangle \\
& +2\left\langle y_{0}, y^{N}(0)\right\rangle-2 \int_{0}^{t} \sigma\left(y^{N}(s), y(s)\right) d s-2 \int_{0}^{t} \sigma\left(y(s), y^{N}(s)\right) d s .
\end{aligned}
$$

Note that

$$
\begin{aligned}
2 \int_{0}^{t}\left\langle\tilde{g}\left(y^{N}(s)\right)-h(s), z^{N}(s)\right\rangle d s & =2 \int_{0}^{t}\left\langle\tilde{g}\left(y^{N}(s)\right)-\tilde{g}(y(s)), z^{N}(s)\right\rangle d s \\
& +2 \int_{0}^{t}\left\langle\tilde{g}(y(s))-h(s), z^{N}(s)\right\rangle d s,
\end{aligned}
$$

and by applying (17) and (11) to (33) we obtain

$$
\begin{aligned}
\left|z^{N}(t)\right|^{2}+2 \int_{0}^{t} k_{1}\left|z^{N}(s)\right|_{\mathcal{V}}^{2} d s & \leq\left|y^{N}(0)-y_{0}\right|^{2}+2(\tilde{L}+\lambda) \int_{0}^{t}\left|z^{N}(s)\right| d s \\
& +\left|X^{N}(t)\right|+\left|Y^{N}(t)\right|
\end{aligned}
$$


where

$$
\begin{aligned}
X^{N}(t) & =2\left[-\left\langle y(t), y^{N}(t)\right\rangle-\int_{0}^{t} \sigma\left(y(s), y^{N}(s)\right) d s-\int_{0}^{t} \sigma\left(y^{N}(s), y(s)\right) d s\right. \\
& +\left\langle y_{0}, y^{N}(0)\right\rangle-\int_{0}^{t}\left\langle\tilde{g}\left(y^{N}(s)\right), y(s)\right\rangle d s-\int_{0}^{t}\left\langle h(s), y^{N}(s)\right\rangle d s \\
& \left.+2 \int_{0}^{t}\langle f(s), y(s)\rangle_{\mathcal{V}^{*}, \mathcal{V}} d s\right], \\
Y^{N}(t) & =2 \int_{0}^{t}\left\langle\tilde{g}(y(s))-h(s), z^{N}(s)\right\rangle d s+2 \int_{0}^{t}\left\langle f(s), z^{N}(s)\right\rangle_{\mathcal{V}^{*}, \mathcal{V}} d s .
\end{aligned}
$$

Note that (25) and (27) imply that $\left|y^{N}(0)-y_{0}\right|^{2} \rightarrow 0$ and $\left|Y^{N}(t)\right| \rightarrow 0$ respectively. It also can be shown that $\left|X^{N}(t)\right| \rightarrow 0$ by taking the limit as $N \rightarrow \infty$ and using the convergences (28), (27), (25) and (29):

$$
\begin{aligned}
X^{N}(t) \rightarrow & 2\left[-|y(t)|^{2}-2 \int_{0}^{t} \sigma(y(s), y(s)) d s+\left|y_{0}\right|^{2}\right. \\
& \left.-2 \int_{0}^{t}\langle h(s), y(s)\rangle d s+2 \int_{0}^{t}\langle f(s), y(s)\rangle_{\mathcal{V}^{*}, \mathcal{V}} d s\right],
\end{aligned}
$$

which is two times the integrated form of (31) with $\psi=y$. Therefore $\left|X^{N}(t)\right| \rightarrow 0$ and (34) becomes

$$
\left|z^{N}(t)\right|^{2}+2 \int_{0}^{t} k_{1}\left|z^{N}(s)\right|_{\mathcal{V}}^{2} d s \leq \hat{L} \int_{0}^{t}\left|z^{N}(s)\right|^{2} d s+W^{N}(t)
$$

where $\hat{L}=2(\tilde{L}+\lambda)$, and where $W^{N}(t)=\left|y^{N}(0)-y_{0}\right|^{2}+\left|X^{N}(t)\right|+\left|Y^{N}(t)\right| \rightarrow 0$ as $N \rightarrow \infty$. Using Gronwall's inequality we obtain

$$
\left|z^{N}(t)\right|^{2} \leq W^{N}(t)+\int_{0}^{t} \hat{L} W^{N}(s) e^{\hat{L} t-\hat{L} s} d s \rightarrow 0
$$

which implies that $\left|z^{N}(t)\right|^{2} \rightarrow 0$ for $t \in[0, T]$ and hence $y^{N}(t) \rightarrow y(t)$ in $\mathcal{H}$ for each $t$. It follows that

$$
\int_{0}^{t}\left|\tilde{g}\left(y^{N}(s)\right)-\tilde{g}(y(s))\right|^{2} d s \leq \tilde{L}^{2} \int_{0}^{t}\left|y^{N}(s)-y(s)\right|^{2} d s \rightarrow 0
$$

so that $\tilde{g}\left(y^{N}\right) \rightarrow \tilde{g}(y)$ in $L_{2}((0, T) ; \mathcal{H})$. Since $(29)$ implies that $\tilde{g}\left(y^{N}\right) \stackrel{w}{\longrightarrow} h$ in $L_{2}((0, T) ; \mathcal{H})$, we have $h=\tilde{g}(y)$ in $L_{2}((0, T) ; \mathcal{H})$ and therefore

$$
\langle h, \psi\rangle=\langle\tilde{g}(y), \psi\rangle
$$

for every $\psi \in \mathcal{V}$. This establishes that $y$ is a solution of (21), (22). The uniqueness of solutions can be shown using standard arguments (see $[3,5]$ ), and is presented in detail in [22]. 
Finally, we show the existence of a solution for the original problem (15), (16) on some interval in time. Since the solution $y$ of $(21),(22)$ satisfies $y \in C([0, T] ; \mathcal{H})$, there exists $t^{*} \in[0, T]$ such that

$$
\left|y(t)-y_{0}\right| \leq 1
$$

for all $t \in\left[0, t^{*}\right]$. Therefore $y(t) \in B_{1}\left(y_{0}\right)$ for all $t \in\left[0, t^{*}\right]$, which further implies that $\tilde{g}(y(t))=g(\mathcal{P} y(t))=g(y(t))$ for all $t \in\left[0, t^{*}\right]$. It follows that $y$ is a solution of (15), (16) on the interval $\left[0, t^{*}\right]$. The uniqueness of the solution $y$ can be shown using standard arguments. This completes the proof of the theorem.

The existence of a unique global solution to (15), (16) now can be shown using the additional assumption (A5).

Theorem 3.2 (Global existence) Under Assumptions (A1) - (A5) with $y_{0} \in \mathcal{H}$, the system (15), (16) has a unique global solution $y \in L_{2}((0, T) ; \mathcal{V}) \cap C([0, T] ; \mathcal{H})$ which depends continuously on the data $\left(y_{0}, f\right)$.

Proof: Let $y^{N}(t)=\sum_{k=1}^{N} c_{k}^{N}(t) \psi_{k}$, where $\left\{c_{k}^{N}(t)\right\}$ are chosen so that $y^{N}(t)$ is a solution of the finite dimensional system of equations derived from (15), (16) with $\psi_{k}$ as test functions. Moreover, we choose the constants $\left\{c_{0 k}^{N}\right\}$ so that

$$
y_{0}=\lim _{N \rightarrow \infty} \sum_{k=1}^{N} y_{0}^{N} \equiv \lim _{N \rightarrow \infty} c_{o k}^{N} \psi_{k} \quad \text { in } \mathcal{V}
$$

As before we obtain

$$
\begin{aligned}
\left|y^{N}(t)\right|^{2}+2 \int_{0}^{t} \sigma\left(y^{N}(s), y^{N}(s)\right) d s & +2 \int_{0}^{t}\left\langle g\left(y^{N}(s)\right), y^{N}(s)\right\rangle d s \\
& =2 \int_{0}^{t}\left\langle f(s), y^{N}(s)\right\rangle_{\mathcal{V}^{*}, \mathcal{V}} d s+\left|y^{N}(0)\right|^{2},
\end{aligned}
$$

and using arguments similar to those for Theorem 3.1 we arrive at the uniform bounds

$$
\left|y^{N}(t)\right|^{2} \leq \hat{C}\left(y_{0}, k_{1}, C_{3}, T, C_{2}, \lambda, f, C_{0}\right)
$$

and

$$
\left|y^{N}(t)\right|^{2}+k_{1} \int_{0}^{t}\left|y^{N}(s)\right|_{\mathcal{V}}^{2} d s \leq \hat{C}_{1}
$$

for $t \in[0, T]$, where $T$ is arbitrary.

This implies that there exists $y \in L_{2}((0, T) ; \mathcal{V})$ and a subsequence of $\left\{y^{N}\right\}$ such that

$$
\begin{aligned}
& y^{N} \stackrel{w}{\rightarrow} y \quad \text { in } \quad L_{2}((0, T) ; \mathcal{V}) \\
& y^{N}(t) \stackrel{w}{\longrightarrow} y(t) \quad \text { a.e. in } \mathcal{H}
\end{aligned}
$$




$$
g\left(y^{N}\right) \stackrel{w}{\longrightarrow} h \quad \text { in } L_{2}((0, T) ; \mathcal{H})
$$

In addition, we use the weak lower semicontinuity of norms to obtain

$$
|y(t)|^{2} \leq \liminf _{N \rightarrow \infty}\left|y^{N}(t)\right|^{2} \leq \hat{C}
$$

for $t \in[0, T]$. Therefore $y(t) \in B_{\sqrt{\hat{C}}}(0)$ in $\mathcal{H}$ for $0 \leq t \leq T$, and the arguments of Theorem 3.1 imply that $y$ is the unique global solution to (15), (16).

To prove continuous dependence, we consider solutions $y$ and $\tilde{y}$ in $L_{2}((0, T) ; \mathcal{V})$ to (15), (16) with initial conditions $y_{0}$ and $\tilde{y}_{0}$ and forcing functions $f$ and $\tilde{f}$, respectively. Note that since $y \in L_{2}((0, T) ; \mathcal{V})$ satisfies $(15)$ with $y(0)=y_{0}$, we can use arguments similar to those used earlier in this proof for $y^{N}(t)$ to obtain the bounds

$$
\begin{aligned}
|y(t)|^{2} \leq \hat{C}\left(y_{0}, k_{1}, C_{3}, T, C_{2}, f, \lambda\right) & =\hat{C}_{2} \\
|\tilde{y}(t)|^{2} & \leq \hat{C}\left(\tilde{y}_{0}, k_{1}, C_{3}, T, C_{2}, \tilde{f}, \lambda\right)=\hat{C}_{3} .
\end{aligned}
$$

Therefore $y(t) \in B_{\sqrt{\hat{C}_{2}}}$ and $\tilde{y}(t) \in B_{\sqrt{\hat{C}_{3}}}$, which imply that

$$
|g(y(t))-g(\tilde{y}(t))| \leq L_{B_{\hat{C}_{4}}}|y(t)-\tilde{y}(t)|,
$$

where $\hat{C}_{4}=\max \left\{\sqrt{\hat{C}_{2}}, \sqrt{\hat{C}_{3}}\right\}$.

Now consider $w(t) \equiv y(t)-\tilde{y}(t)$, which satisfies $w(0)=y_{0}-\tilde{y}_{0}$ and

$$
\langle\dot{w}(t), \psi\rangle_{\mathcal{V}^{*}, \mathcal{V}}+\sigma(w(t), \psi)+\langle\Delta g(t), \psi\rangle=\langle\hat{f}(t), \psi\rangle_{\mathcal{V}^{*}, \mathcal{V}}
$$

for all $\psi \in \mathcal{V}$, where $\Delta g(t)=g(y(t))-g(\tilde{y}(t))$ and $\hat{f}(t)=f(t)-\tilde{f}(t)$. Note that (39) is also satisfied for all $\psi \in L_{2}((0, T) ; \mathcal{V})$.

For fixed $t \in[0, T]$ we choose $\psi=w(t) \in \mathcal{V}$ in (39) and integrate from 0 to $t$ :

$$
\int_{0}^{t}\left[\langle\dot{w}(s), w(s)\rangle_{\mathcal{V}^{*}, \mathcal{V}}+\sigma(w(s), w(s))+\langle\Delta g(s), w(s)\rangle\right] d s=\int_{0}^{t}\langle\hat{f}(s), w(s)\rangle_{\mathcal{V}^{*}, \mathcal{V}} d s .
$$

We then apply the conditions (11), (38) and Gronwall's inequality to obtain

$$
|w(t)|^{2} \leq e^{2\left(L_{B_{\hat{C}_{4}}}+\lambda\right) T}\left(|w(0)|^{2}+\frac{1}{k_{1}} \int_{0}^{T}|\hat{f}(s)|_{\mathcal{V}^{*}}^{2} d s\right)
$$

for all $t \in[0, T]$. Therefore we have shown continuous dependence in $C([0, T] ; \mathcal{H})$ on the initial data $\left(y_{0}, f\right)$. This completes the proof of Theorem 3.2.

\subsection{Well-posedness for the TCE model}

In this section we verify that the TCE model fits into the class of abstract nonlinear parabolic systems discussed in the previous section. This will establish the wellposedness of the TCE model. 
Consider the weak formulation (2) of the TCE model with the definitions of $\mathcal{V}$, $\mathcal{H}$ and the corresponding norms and inner products as outlined in Section 2.1. The spaces $\mathcal{V}$ and $\mathcal{H}$ form a Gelfand triple as discussed in Section 3.1 with the duality product $\langle\cdot, \cdot\rangle_{\mathcal{V}^{*}, \mathcal{V}}$. It can be shown using standard arguments that Assumptions (A1) - (A5) are satisfied for the TCE PBPK-hybrid weak formulation (see [22] for detailed proofs). Therefore we can apply Theorem 3.2 to establish the existence, uniqueness and continuous dependence of a global solution for the TCE model given by $(2)-(5)$.

\section{Estimation of parameters and convergence of Galerkin approximations}

The TCE PBPK-hybrid model is dependent on many physical, biological and chemical parameters, some of which can be explicitly measured. However, most of the adipose model parameters are unknown, and it would be impractical to measure them experimentally. Therefore, we must use inverse problem techniques to estimate these parameters.

In this section we address theoretical issues related to the standard parameter estimation problem associated with the TCE PBPK-hybrid model, and we establish the theoretical convergence of our numerical approximation scheme. The results we present here are based on the work of Banks and Kunisch [4], who developed results for a general parameter estimation problem. Banks and Musante [6] applied these results to the abstract class of nonlinear parabolic systems discussed in [5]. Here we extend the ideas presented in [4] and [6] to the TCE model and the abstract class of nonlinear parabolic systems discussed in Section 3.

Consider the abstract problem

$$
\begin{aligned}
& \dot{y}(t)+\mathcal{A}(q) y(t)+g(q)(y(t))=f(t ; q) \\
& y(0)=y_{0}
\end{aligned}
$$

which is a parameterized formulation of the system (7), (8). Specifically, the sesquilinear form $\sigma$, the nonlinearity $g$ and the forcing function $f$ are now functions of a vector $q$ of parameters which must be estimated using data.

The parameter vector $q$ belongs to a set $Q$ of admissible parameters, where $Q$ may be an infinite dimensional space. In the case of our TCE model, the physiology of adipose tissue (see [2, 22]) suggests that many of the adipose model parameters may be dependent on time and/or space. This includes parameters such as the 
volumetric blood flow rate $Q_{f}$ to adipose tissue and the permeability coefficients $\mu_{B A}, \mu_{B I}$ and $\mu_{I A}$. Such a dependency on time and/or space would lead to infinite dimensional parameters and an infinite dimensional parameter space $Q$.

Using arguments and modified conditions as outlined in Section 3, we can readily establish the existence of a unique solution to the system

$$
\begin{aligned}
& \langle\dot{y}(t), \psi\rangle_{\mathcal{V}^{*}, \mathcal{V}}+\langle\mathcal{A}(q) y(t), \psi\rangle_{\mathcal{V}^{*}, \mathcal{V}}+\langle g(q)(y(t)), \psi\rangle=\langle f(t ; q), \psi\rangle_{\mathcal{V}^{*}, \mathcal{V}} \\
& y(0)=y_{0}
\end{aligned}
$$

for $\psi \in \mathcal{V}$. Specifically, the modified conditions used to prove the existence of a solution to the parameterized system (42), (43) include the extra requirement of uniformity with respect to the parameters $q$.

These solutions $y(\cdot, q)$ to the parameterized problem can be used with experimental data to estimate the parameters $q$ by solving the least squares parameter estimation problem given by

$$
\min _{q \in Q} J(q, z)=\sum_{i=1}^{N_{t}}\left|\mathcal{O} y\left(t_{i}, \cdot ; q\right)-z_{i}\right|^{2},
$$

where $z_{i}, i=1, \ldots, N_{t}$ are observations taken at time $t_{i}$, and $|\cdot|$ is an appropriately chosen Euclidean norm. The solutions $y\left(t_{i}, \cdot ; q\right)$ belong to an infinite dimensional state space $\mathcal{H}$. As discussed in [4], the observation operator $\mathcal{O}$ is determined by the type of data that is being collected. Examples appropriate for the TCE model and related experiments include observations of concentrations taken in time at a given point, or observations in time of an average concentration over a region. This latter type of observation may be used when concentrations are measured from homogenized tissue samples.

As in $[4,6,7]$, we address the parameter estimation problem (44) by studying the convergence properties of the finite dimensional approximating parameter estimation problems

$$
\min _{q \in Q^{M}} J^{N}(q)=\sum_{i=1}^{N_{t}}\left|\mathcal{O} y^{N}\left(t_{i}, \cdot ; q\right)-z_{i}\right|^{2},
$$

where the sets $Q^{M}$ are a sequence of finite dimensional sets that approximate $Q$, and $y^{N}(t) \in \mathcal{H}^{N} \subset \mathcal{H}$ is the solution to the following finite dimensional approximation of (42), (43):

$$
\begin{aligned}
& \left\langle\dot{y}^{N}(t), \psi\right\rangle+\left\langle\mathcal{A}(q) y^{N}(t), \psi\right\rangle_{\mathcal{V}^{*}, \mathcal{V}}+\left\langle g(q)\left(y^{N}(t)\right), \psi\right\rangle=\langle f(t ; q), \psi\rangle_{\mathcal{V}^{*}, \mathcal{V}} \\
& y^{N}(0)=P^{N} y_{0}
\end{aligned}
$$

for $\psi \in \mathcal{H}^{N}$. The set $\mathcal{H}^{N}$ is a finite dimensional subspace of the state space $\mathcal{H}$, and $P^{N}$ is the orthogonal projection of $\mathcal{H}^{N}$ onto $\mathcal{H}$. 
Assuming certain compactness conditions that we detail below, we are guaranteed that for each $M$ and $N$ there exists a minimizer $\bar{q}^{M, N}$ for the finite dimensional estimation problem (45). In this section we address the convergence properties of this sequence of minimizers as $M, N \rightarrow \infty$, and the circumstances for which we can guarantee convergence to a minimizer for the infinite dimensional parameter estimation problem (44). The results we prove below for the abstract class of systems in Section 3 are adaptations and extensions of results for the general estimation problem discussed in [4] and the estimation problem for the class of nonlinear parabolic systems presented in [6]. We also show that the results established here apply specifically to the TCE PBPK-hybrid model, which is a member of the class of systems described above.

We obtain as a special case (with $q^{N} \equiv q$ fixed in Theorem 4.3 below) the convergence of solutions for the finite dimensional approximations (46), (47) to the solution for the infinite dimensional system (42), (43). This establishes the convergence of the numerical scheme that we implement computationally in [22] to generate model simulations and for use in parameter estimation problems.

\subsection{Well-posedness of solutions for the parameterized system}

In this section we address the well-posedness of solutions for (40), (41). That is, using the arguments detailed in Section 3, we may prove for this abstract system the existence of a unique solution which depends continuously on the initial data.

As in Section 3.1, we define the state spaces $\mathcal{V}$ and $\mathcal{H}$ which form the Gelfand triple

$$
\mathcal{V} \hookrightarrow \mathcal{H} \hookrightarrow \mathcal{V}^{*},
$$

where $\mathcal{V}$ is continuously and densely embedded in $\mathcal{H}$. Moreover, there exists $k>0$ such that

$$
|\psi| \leq k|\psi|_{\mathcal{V}}
$$

for all $\psi \in \mathcal{V}$, where $|\cdot|_{\mathcal{V}}$ is the norm on $\mathcal{V}$ and $|\cdot|$ denotes the norm on $\mathcal{H}$. We continue to use the notation $\langle\cdot, \cdot\rangle_{\mathcal{V}^{*}, \mathcal{V}}$ to represent the duality product, which is the extension by continuity of the inner product $\langle\cdot, \cdot\rangle$ in $\mathcal{H}$ (see Section 3.1).

Here we consider the system

$$
\begin{aligned}
& \langle\dot{y}(t), \psi\rangle_{\mathcal{V}^{*}, \mathcal{V}}+\sigma(q)(y(t), \psi)+\langle g(q)(y(t)), \psi\rangle=\langle f(t ; q), \psi\rangle_{\mathcal{V}^{*}, \mathcal{V}} \\
& y(0)=y_{0}
\end{aligned}
$$

for $\psi \in \mathcal{V}$, where the sesquilinear form $\sigma(q): \mathcal{V} \times \mathcal{V} \rightarrow \mathbb{R}$ is associated with the operator $\mathcal{A}(q): \mathcal{V} \rightarrow \mathcal{V}^{*}$ so that $\sigma(q)(u, v)=\langle\mathcal{A}(q) u, v\rangle_{\mathcal{V}^{*}, \mathcal{V}}$ for all $u, v \in \mathcal{V}$. 
We make the following assumptions for the parameterized system, which are the same assumptions as those made in Section 3.1 with the extra requirement of uniformity in $q \in Q$ :

(A1') The sesquilinear form $\sigma(q)$ is uniformly bounded in $\mathcal{V}$, i.e., there exists $C_{1}>0$ independent of $q \in Q$ such that

$$
|\sigma(q)(u, v)| \leq C_{1}|u|_{\mathcal{V}}|v|_{\mathcal{V}} \quad \text { for all } u, v \in \mathcal{V} .
$$

$\left(\mathrm{A} 2^{\prime}\right)$ The sesquilinear form $\sigma(q)$ is uniformly strictly coercive on $\mathcal{V}$. That is, there exist $k_{1}, \lambda>0$ independent of $q \in Q$ such that

$$
\sigma(q)(u, u) \geq k_{1}|u|_{\mathcal{V}}^{2}-\lambda|u|^{2} \quad \text { for all } u \in \mathcal{V} .
$$

$\left(\mathrm{A} 3^{\prime}\right)$ The forcing function $f(\cdot ; q)$ satisfies

$$
f(\cdot ; q) \in L_{2}\left((0, T) ; \mathcal{V}^{*}\right)
$$

for each $q \in Q$.

(A4') The nonlinear function $g(q): \mathcal{H} \rightarrow \mathcal{H}$ satisfies the following uniform local Lipschitz condition: let $B_{r}(0)=\{u \in \mathcal{H}:|u| \leq r\}$ denote the ball of radius $r$ centered around the origin in $\mathcal{H}$. Then given $r>0$, there exists $L_{B_{r}}>0$ independent of $q \in Q$ such that

$$
|g(q)(u)-g(q)(v)| \leq L_{B_{r}}|u-v| \quad \text { for all } u, v \in B_{r}(0) .
$$

$\left(\mathrm{A} 5^{\prime}\right)$ There exist positive constants $C_{2}$ and $C_{3}$ independent of $q \in Q$ such that

$$
|g(q)(u)| \leq C_{2}|u|+C_{3} \quad \text { for all } u \in \mathcal{H}
$$

Under these assumptions we can prove the existence of a unique solution to the parameterized system (49), (50). The proof of this theorem is similar to the proof for Theorem 3.2 .

Theorem 4.1 Under Assumptions $\left(A 1^{\prime}\right)-\left(A 5^{\prime}\right)$ and for any $y_{0} \in \mathcal{H}$, the system (49) - (50) has a unique global solution $y \in L_{2}((0, T) ; \mathcal{V}) \cap C([0, T] ; \mathcal{H})$ which depends continuously on the initial data, with $\dot{y} \in L_{2}\left((0, T) ; \mathcal{V}^{*}\right)$.

\subsection{The general parameter estimation problem}

In this section we present theorems related to convergence for the general parameter estimation problem

$$
\min _{q \in Q} J(q, z)=\sum_{i=1}^{N_{t}}\left|\mathcal{O} y\left(t_{i}, \cdot ; q\right)-z_{i}\right|^{2}
$$


given the observations $z_{i}, i=1, \ldots, N_{t}$ taken at time $t_{i}$ and the observation operator $\mathcal{O}$. Moreover, $y\left(t_{i}, \cdot ; q\right)$ are the solutions of the parameterized system (49), (50). As in Section 3.1, we use Galerkin type approximations applied to the parameterized system (40), (41).

Proceeding as in $[6,7]$, we make the following assumptions about the state and parameter spaces $\mathcal{H}, Q, \mathcal{H}^{N}$ and $Q^{M}$ :

(B1) The sets $Q$ and $Q^{M}$ each lie in a metric space $\tilde{Q}$ with metric $d$. We assume that $Q$ and $Q^{M}$ are compact in this metric, and that there is a mapping $i^{M}: Q \rightarrow Q^{M}$ such that $Q^{M}=i^{M}(Q)$. Moreover, for each $q \in Q$ we have $i^{M}(q) \rightarrow q$ in $Q$, where the convergence is uniform in $q \in Q$.

(B2) The finite dimensional subspaces $\mathcal{H}^{N}$ satisfy $\mathcal{H}^{N} \subset \mathcal{V}$ for all $N$.

(B3) For each $\psi \in \mathcal{V},\left|\psi-P^{N} \psi\right|_{\mathcal{V}} \rightarrow 0$ as $N \rightarrow \infty$, where $P^{N}$ is the orthogonal projection operator of $\mathcal{H}$ onto $\mathcal{H}^{N}$.

Moreover, we assume that $\mathcal{A}(q), g(q)$ and $f(\cdot ; q)$ depend continuously on the parameters $q \in Q$ in the sense that

(C1) $|\sigma(q)(u, v)-\sigma(\tilde{q})(u, v)| \leq d_{1}(q, \tilde{q})|u|_{\mathcal{V}}|v|_{\mathcal{V}}$ for all $u, v \in \mathcal{V}$, where $d_{1}(q, \tilde{q}) \rightarrow 0$ as $d(q, \tilde{q}) \rightarrow 0$.

(C2) $|g(q)(u)-g(\tilde{q})(u)| \leq d_{2}(q, \tilde{q})|u|$ for all $u \in \mathcal{H}$, where $d_{2}(q, \tilde{q}) \rightarrow 0$ as $d(q, \tilde{q}) \rightarrow$ 0.

(C3) The mapping $q \rightarrow f(\cdot ; q)$ is continuous from $Q$ to $L_{2}\left((0, T) ; \mathcal{V}^{*}\right)$.

Note that the compactness conditions of Assumption (B1) guarantee the existence of a minimizer $\bar{q}^{M, N}$ to the finite dimensional estimation problem (45). The following theorem from [7] (Theorem 5.1, p. 124) establishes conditions for which the sequence of minimizers $\bar{q}^{M, N}$ converges to a minimizer for the infinite dimensional estimation problem (44).

Theorem 4.2 To obtain convergence of at least a subsequence of $\left\{\bar{q}^{M, N}\right\}$ to a solution $\bar{q}$ of minimizing (44) subject to (42), (43), it suffices, under Assumption (B1), to argue that for arbitrary sequences $\left\{q^{M, N}\right\}$ in $Q$ with $q^{M, N} \rightarrow q$ in $Q$, we have

$$
\mathcal{O} y^{N}\left(t ; q^{M, N}\right) \rightarrow \mathcal{O} y(t ; q) .
$$

The condition (56) implies that the original sequence of Galerkin approximations in Section 3.1 converges, which is stronger than the subsequential convergence 
proven there. This stronger result is important for establishing the convergence of the numerical scheme that we develop and implement in [22].

Now we verify the conditions of Theorem 4.2 for the general estimation problem described in this section.

Theorem 4.3 Assume that Assumptions (B1) - (B3) and (C1) - (C3) are satisfied, in addition to the conditions of Theorem 4.1. Let $q^{N}$ be any sequence in $Q^{N}$ satisfying $q^{N} \rightarrow q \in Q$. Then

$$
\begin{aligned}
& y^{N}\left(t ; q^{N}\right) \rightarrow y(t ; q) \quad \text { in } \mathcal{H} \text { uniformly on }[0, T], \\
& y^{N}\left(t ; q^{N}\right) \rightarrow y(t ; q) \quad \text { in } \mathcal{V} \text { for almost all } t>0,
\end{aligned}
$$

where $y^{N}$ satisfies

$$
\begin{aligned}
& \left\langle\dot{y}^{N}(t), \psi\right\rangle+\sigma\left(q^{N}\right)\left(y^{N}(t), \psi\right)+\left\langle g\left(q^{N}\right)\left(y^{N}(t)\right), \psi\right\rangle=\left\langle f\left(t ; q^{N}\right), \psi\right\rangle_{\mathcal{V}^{*}, \mathcal{V}} \\
& y^{N}(0)=P^{N} y_{0}
\end{aligned}
$$

for all $\psi \in \mathcal{H}^{N}$, and y satisfies (49) - (50) for all $\psi \in \mathcal{V}$.

Proof: It follows from Theorem 4.1 that $y(t) \in \mathcal{H}$ for every $t \in[0, T]$ and $y(t) \in \mathcal{V}$ for almost every $t \in[0, T]$. Moreover,

$$
\left|y^{N}\left(t ; q^{N}\right)-y(t ; q)\right| \leq\left|y^{N}\left(t ; q^{N}\right)-P^{N} y(t ; q)\right|+\left|P^{N} y(t ; q)-y(t ; q)\right| .
$$

By Assumption (B3), we have $\left|\psi-P^{N} \psi\right|_{\mathcal{V}} \rightarrow 0$ for each $\psi \in \mathcal{V}$ as $N \rightarrow \infty$. Note that $y \in C([0, T] ; \mathcal{H})$ implies that $\{y(t): t \in[0, T]\}$ is compact in $\mathcal{H}$, which along with (48) and the dense embedding $\mathcal{V} \hookrightarrow \mathcal{H}$ further implies that

$$
\left|P^{N} y(t ; q)-y(t ; q)\right| \rightarrow 0
$$

uniformly in $t \in[0, T]$. Therefore it suffices to show that

$$
\left|y^{N}\left(t ; q^{N}\right)-P^{N} y(t ; q)\right| \rightarrow 0
$$

uniformly on $[0, T]$ as $N \rightarrow \infty$.

As in [6], we define

$$
\begin{aligned}
y^{N} & =y^{N}\left(t ; q^{N}\right) \\
y & =y(t ; q) \\
\Delta^{N} & =y^{N}\left(t ; q^{N}\right)-P^{N} y(t ; q)=y^{N}-P^{N} y .
\end{aligned}
$$

It follows that

$$
\dot{\Delta}^{N}=\dot{y}^{N}-\frac{d}{d t} P^{N} y,
$$


and hence for all $\psi \in \mathcal{H}^{N}$ we have

$$
\begin{aligned}
\left\langle\dot{\Delta}^{N}, \psi\right\rangle_{\mathcal{V}^{*}, \mathcal{V}} & =\left\langle\dot{y}^{N}-\frac{d}{d t} P^{N} y, \psi\right\rangle_{\mathcal{V}^{*}, \mathcal{V}} \\
& =\left\langle\dot{y}^{N}-\dot{y}+\dot{y}-\frac{d}{d t} P^{N} y, \psi\right\rangle_{\mathcal{V}^{*}, \mathcal{V}} \\
& =\left\langle\dot{y}^{N}, \psi\right\rangle-\langle\dot{y}, \psi\rangle_{\mathcal{V}^{*}, \mathcal{V}}+\left\langle\dot{y}-\frac{d}{d t} P^{N} y, \psi\right\rangle_{\mathcal{V}^{*}, \mathcal{V}}
\end{aligned}
$$

Moreover, we use (49) and (57) to obtain

$$
\begin{aligned}
\left\langle\dot{\Delta}^{N}, \psi\right\rangle_{\mathcal{V}^{*}, \mathcal{V}} & =\left\langle\dot{y}-\frac{d}{d t} P^{N} y, \psi\right\rangle_{\mathcal{V}^{*}, \mathcal{V}}+\left\langle f\left(t ; q^{N}\right)-f(t ; q), \psi\right\rangle_{\mathcal{V}^{*}, \mathcal{V}} \\
& +\sigma\left(q^{N}\right)\left(y-P^{N} y, \psi\right)+\sigma(q)(y, \psi)-\sigma\left(q^{N}\right)(y, \psi) \\
& +\left\langle g(q)(y)-g\left(q^{N}\right)\left(y^{N}\right), \psi\right\rangle-\sigma\left(q^{N}\right)\left(y^{N}-P^{N} y, \psi\right)
\end{aligned}
$$

for all $\psi \in \mathcal{H}^{N}$, where we have added and subtracted $\sigma\left(q^{N}\right)\left(y-P^{N} y, \psi\right)$. It then follows from the definition of $\Delta^{N}$ that

$$
\begin{aligned}
\left\langle\dot{\Delta}^{N}, \psi\right\rangle_{\mathcal{V}^{*}, \mathcal{V}}+ & \sigma\left(q^{N}\right)\left(\Delta^{N}, \psi\right)=\left\langle\dot{y}-\frac{d}{d t} P^{N} y, \psi\right\rangle_{\mathcal{V}^{*}, \mathcal{V}}+\left\langle f\left(t ; q^{N}\right)-f(t ; q), \psi\right\rangle_{\mathcal{V}^{*}, \mathcal{V}} \\
& +\sigma(q)(y, \psi)-\sigma\left(q^{N}\right)(y, \psi)+\sigma\left(q^{N}\right)\left(y-P^{N} y, \psi\right) \\
& +\left\langle g(q)(y)-g\left(q^{N}\right)\left(y^{N}\right), \psi\right\rangle
\end{aligned}
$$

for all $\psi \in \mathcal{H}^{N}$. Now we set $\psi=\Delta^{N} \in \mathcal{H}^{N}$ in (59) to arrive at the equation

$$
\begin{aligned}
\frac{1}{2} \frac{d}{d t}\left[\left|\Delta^{N}\right|^{2}\right]+ & \sigma\left(q^{N}\right)\left(\Delta^{N}, \Delta^{N}\right)=\left\langle\dot{y}-\frac{d}{d t} P^{N} y, \Delta^{N}\right\rangle_{\mathcal{V}^{*}, \mathcal{V}} \\
& +\left\langle f\left(t ; q^{N}\right)-f(t ; q), \Delta^{N}\right\rangle_{\mathcal{V}^{*}, \mathcal{V}}+\sigma(q)\left(y, \Delta^{N}\right)-\sigma\left(q^{N}\right)\left(y, \Delta^{N}\right) \\
& +\sigma\left(q^{N}\right)\left(y-P^{N} y, \Delta^{N}\right)+\left\langle g(q)(y)-g\left(q^{N}\right)\left(y^{N}\right), \Delta^{N}\right\rangle
\end{aligned}
$$

for almost all $t \in[0, T]$. We integrate the left side of (60) and apply Assumption $\left(\mathrm{A} 2^{\prime}\right)$ and the initial condition

$$
\Delta^{N}(0)=y^{N}(0)-P^{N} y(0)=y^{N}(0)-P^{N} y_{0}=0
$$

to obtain the inequality

$$
\begin{aligned}
\int_{0}^{t} \frac{1}{2} \frac{d}{d t}\left|\Delta^{N}(s)\right|^{2} d s & +\int_{0}^{t} \sigma\left(q^{N}\right)\left(\Delta^{N}(s), \Delta^{N}(s)\right) d s \\
& \geq \frac{1}{2}\left|\Delta^{N}(t)\right|^{2}+k_{1} \int_{0}^{t}\left|\Delta^{N}(s)\right|_{\mathcal{V}}^{2} d s-\lambda \int_{0}^{t}\left|\Delta^{N}(s)\right|^{2} d(61)
\end{aligned}
$$

for all $t \in[0, T]$.

Next we integrate the right side of (60) from 0 to $t$. Note that the first term is equal to zero, which follows from the fact that $P^{N}$ is the orthogonal projection from $\mathcal{H}$ onto $\mathcal{H}^{N}$. Indeed, since $y-P^{N} y \in \mathcal{H}$ we have

$$
\left\langle\dot{y}-\frac{d}{d t} P^{N} y, \psi\right\rangle_{\mathcal{V}^{*}, \mathcal{V}}=\frac{d}{d t}\left\langle y-P^{N} y, \psi\right\rangle=0
$$


for all $\psi \in \mathcal{H}^{N}$ since $P^{N}$ is the orthogonal projection of $\mathcal{H}$ onto $\mathcal{H}^{N}$, which implies that $\left(I-P^{N}\right) y$ is orthogonal to all elements in $\mathcal{H}^{N}$ (including $\Delta^{N}(s)$ for all $s \in$ $[0, T])$.

Integrating the second term on the right side of (60), we use the inequality $a b \leq(1 / 2) a^{2}+(1 / 2) b^{2}$ to obtain

$$
\begin{aligned}
\int_{0}^{t}\left\langle f\left(s ; q^{N}\right)-f(s ; q), \Delta^{N}(s)\right\rangle_{\mathcal{V}^{*}, \mathcal{V}} d s & \leq \frac{1}{2 \beta} \int_{0}^{t}\left|f\left(s ; q^{N}\right)-f(s ; q)\right|_{\mathcal{V}^{*}}^{2} d s \\
& +\frac{\beta}{2} \int_{0}^{t}\left|\Delta^{N}(s)\right|_{\mathcal{V}}^{2} d s
\end{aligned}
$$

where $\beta>0$. We proceed in a similar manner with the third and fourth terms on the right side of (60), applying Assumption (C1):

$$
\begin{aligned}
\int_{0}^{t}\left[\sigma(q)\left(y(s), \Delta^{N}(s)\right)-\sigma\left(q^{N}\right)\left(y(s), \Delta^{N}(s)\right)\right] d s & \leq \frac{d_{1}^{2}\left(q, q^{N}\right)}{2 \beta} \int_{0}^{t}|y(s)|_{\mathcal{V}}^{2} d s \\
& +\frac{\beta}{2} \int_{0}^{t}\left|\Delta^{N}(s)\right|_{\mathcal{V}}^{2} d s .
\end{aligned}
$$

The integral of the fifth term on the right side of (60) can be bounded using Assumption $\left(\mathrm{A} 1^{\prime}\right)$ :

$$
\begin{aligned}
\int_{0}^{t} \sigma\left(q^{N}\right)\left(y(s)-P^{N} y(s), \Delta^{N}(s)\right) d s & \leq \frac{C_{1}^{2}}{2 \beta} \int_{0}^{t}\left|y(s)-P^{N} y(s)\right|_{\mathcal{V}}^{2} d s \\
& +\frac{\beta}{2} \int_{0}^{t}\left|\Delta^{N}(s)\right|_{\mathcal{V}}^{2} d s .
\end{aligned}
$$

Finally, we integrate the sixth term from 0 to $t$, obtaining

$$
\begin{aligned}
\int_{0}^{t}\left\langle g(q)(y(s))-g\left(q^{N}\right)\right. & \left.\left(y^{N}(s)\right), \Delta^{N}(s)\right\rangle d s \\
\leq & \int_{0}^{t}\left|\left\langle g(q)(y(s))-g\left(q^{N}\right)(y(s)), \Delta^{N}(s)\right\rangle\right| d s \\
& \quad+\int_{0}^{t}\left|\left\langle g\left(q^{N}\right)(y(s))-g\left(q^{N}\right)\left(y^{N}(s)\right), \Delta^{N}(s)\right\rangle\right| d s .
\end{aligned}
$$

The first term on the right side of (66) can be estimated using Assumption (C2) and (48):

$$
\begin{aligned}
\int_{0}^{t} \mid\langle g(q)(y(s))- & \left.g\left(q^{N}\right)(y(s)), \Delta^{N}(s)\right\rangle\left.\left|d s \leq \int_{0}^{t} d_{2}\left(q, q^{N}\right) k^{2}\right| y(s)\right|_{\mathcal{V}}\left|\Delta^{N}(s)\right|_{\mathcal{V}} d s \\
\leq & \frac{k^{2} d_{2}^{2}\left(q, q^{N}\right)}{2 \beta} \int_{0}^{t}|y(s)|_{\mathcal{V}}^{2} d s+\frac{k^{2} \beta}{2} \int_{0}^{t}\left|\Delta^{N}(s)\right|_{\mathcal{V}}^{2} d s
\end{aligned}
$$

We obtain a bound for the second term on the right side of (66) using Assumption $\left(\mathrm{A} 4^{\prime}\right)$ applied to $y(s)$ and $P^{N} y(s)$. In the proof of Theorem 3.2, we showed that the solution $y \in L_{2}((0, T) ; \mathcal{V}) \cap C([0, T] ; \mathcal{H})$ to the system $(15),(16)$ is bounded by the constant $\hat{C}$ as in (37). It is easily seen that for each $s \in[0, T]$, the same bound

$$
|y(s)|^{2} \leq \hat{C}
$$


applies for the solution $y \in L_{2}((0, T) ; \mathcal{V}) \cap C([0, T] ; \mathcal{H})$ to the system $(49),(50)$ which is guaranteed by Theorem 4.1. Moreover, for each $s \in[0, T]$ we have

$$
\left|P^{N} y(s)\right| \leq\left\|P^{N}\right\||y(s)|=|y(s)|,
$$

which implies that both $y(s)$ and $P^{N} y(s)$ are in the ball $B \sqrt{\hat{C}}$ in $\mathcal{H}$. Using the same arguments and the bound (36), we can show that $y^{N}(s)$ is in $B \sqrt{\hat{C}}$. Hence we may apply Assumption (A4') and (48) to obtain

$$
\begin{aligned}
\int_{0}^{t} \mid\left\langle g\left(q^{N}\right)(y(s))-\right. & \left.g\left(q^{N}\right)\left(y^{N}(s)\right), \Delta^{N}(s)\right\rangle \mid d s \\
& \leq \int_{0}^{t}\left|\left\langle g\left(q^{N}\right)(y(s))-g\left(q^{N}\right)\left(P^{N} y(s)\right), \Delta^{N}(s)\right\rangle\right| d s \\
& +\int_{0}^{t}\left|\left\langle g\left(q^{N}\right)\left(P^{N} y(s)\right)-g\left(q^{N}\right)\left(y^{N}(s)\right), \Delta^{N}(s)\right\rangle\right| d s \\
& \leq \frac{k^{2} L_{B}^{2}}{2 \beta} \int_{0}^{t}\left|y(s)-P^{N} y(s)\right|_{\mathcal{V}}^{2} d s+\frac{k^{2} \beta}{2} \int_{0}^{t}\left|\Delta^{N}(s)\right|_{\mathcal{V}}^{2} d s \\
& +L_{B} \sqrt{\widehat{c}} \int_{0}^{t}\left|\Delta^{N}(s)\right|^{2} d s .
\end{aligned}
$$

Now we combine the terms in (61) - (68) and use (60) to arrive at the estimate

$$
\begin{aligned}
& \frac{1}{2}\left|\Delta^{N}(t)\right|^{2}+\left(k_{1}-\left(3+2 k^{2}\right) \frac{\beta}{2}\right) \int_{0}^{t}\left|\Delta^{N}(s)\right|_{\mathcal{V}}^{2} d s \\
& \leq \frac{1}{2 \beta} \int_{0}^{t}\left|f\left(s ; q^{N}\right)-f(s ; q)\right|_{\mathcal{V}^{*}}^{2} d s+\frac{d_{1}^{2}\left(q, q^{N}\right)}{2 \beta} \int_{0}^{t}|y(s)|_{\mathcal{V}}^{2} d s \\
& +\frac{C_{1}^{2}}{2 \beta} \int_{0}^{t}\left|y(s)-P^{N} y(s)\right|_{\mathcal{V}}^{2} d s+\frac{k^{2} d_{2}^{2}\left(q, q^{N}\right)}{2 \beta} \int_{0}^{t}|y(s)|_{\mathcal{V}}^{2} d s \\
& +\frac{k^{2} L_{B}^{2} \sqrt{c}}{2 \beta} \int_{0}^{t}\left|y(s)-P^{N} y(s)\right|_{\mathcal{V}}^{2} d s+\left(L_{B \sqrt{c}}+\lambda\right) \int_{0}^{t}\left|\Delta^{N}(s)\right|^{2} d s \\
& \equiv \delta^{N}(t)+\left(L_{B \sqrt{c}}+\lambda\right) \int_{0}^{t}\left|\Delta^{N}(s)\right|^{2} d s \\
& \leq \delta^{N}(T)+\left(L_{B \sqrt{C}}+\lambda\right) \int_{0}^{t}\left|\Delta^{N}(s)\right|^{2} d s
\end{aligned}
$$

for $t \in[0, T]$. Note that as $N \rightarrow \infty$ we have $\delta^{N}(t) \rightarrow 0$ for each $t \in[0, T]$. This is a consequence of Assumption (C3), $q^{N} \rightarrow q, y \in L_{2}((0, T) ; \mathcal{V})$, and Assumption (B3).

Now we choose $\beta>0$ in (69) so that $k_{1}-\left(3+2 k^{2}\right) \frac{\beta}{2}>0$. We then apply Gronwall's inequality to obtain

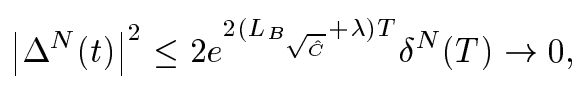

which implies that $\Delta^{N} \rightarrow 0$ in $C([0, T] ; \mathcal{H})$ as $N \rightarrow \infty$. Moreover, combining (69) with (70) we have

$$
\left(k_{1}-\left(3+2 k^{2}\right) \frac{\beta}{2}\right) \int_{0}^{t}\left|\Delta^{N}(s)\right|_{\mathcal{V}}^{2} d s \leq \delta^{N}(T)+\left(L_{B \sqrt{\widehat{C}}}+\lambda\right) \int_{0}^{t}\left|\Delta^{N}(s)\right|^{2} d s
$$




$$
\begin{aligned}
& \leq \delta^{N}(T)\left(1+2 T\left(L_{B}{ }_{\sqrt{C}}+\lambda\right) e^{2\left(L_{B} \sqrt{\widehat{C}}+\lambda\right) T}\right) \\
& \rightarrow 0
\end{aligned}
$$

and hence $\Delta^{N} \rightarrow 0$ in $L_{2}((0, T) ; \mathcal{V})$. This completes the proof of the theorem.

\subsection{Parameter estimation for the TCE model}

The TCE PBPK-hybrid

model outlined in Section 2 includes several physical parameters, each of which is considered a positive constant that is bounded above by some maximum admissible value. This implies that the parameters for this model lie in a compact subset of Euclidean space. Therefore we set $Q^{M}=Q$ for all $M$.

Using arguments detailed in [22], it can be shown that Assumptions (A1') $\left(\mathrm{A} 5^{\prime}\right)$ are satisfied for the TCE PBPK-hybrid model. We state without proof that Assumptions (C1) - (C3) also are satisfied for the TCE model. Moreover, it is seen that Assumptions (B1) - (B3) hold for our choice of state spaces $\mathcal{V}, \mathcal{H}$ and $\mathcal{H}^{N}$ defined in Section 2.1. It follows that the theoretical results presented in Section 4.2 apply to the TCE PBPK-hybrid model.

Experimental observations collected in the experiments of Evans et al. [15] include measurements of TCE concentrations in the venous blood, as well as unbound concentrations in the fat, liver and brain tissue. The tissue concentrations are collected from homogenized tissue samples, leading to a measurement of an effective average tissue concentration. For the liver and brain tissues our model assumes well-mixed compartments, which implies that the average tissue concentration is equal to the concentration at any point in that tissue. Therefore the observation operators for the venous blood and the brain and liver tissues are given by

$$
\mathcal{O} y\left(t_{i}, \cdot ; q\right)=C_{j}\left(t_{i} ; q\right)
$$

where $C_{j}=C_{v}, C_{b r}$ and $C_{l}$ respectively.

In the adipose tissue our model assumes spatially-varying concentrations, so that an effective average concentration is calculated by taking an average of concentrations over the adipocyte region. This implies an observation operator of the form

$$
\mathcal{O} y\left(t_{i}, \cdot ; q\right)=\frac{1}{2 \pi^{2}} \int_{0}^{\pi} \int_{0}^{2 \pi} C_{A}\left(t_{i}, \theta, \phi\right) d \theta d \phi .
$$

Each of these observation operators is utilized in [22], where we present detailed computational parameter estimation results for the TCE model. 


\section{Concluding remarks}

In this paper we have addressed theoretical issues related to the TCE PBPK-hybrid model and a general class of toxicokinetic models. Specifically, we established existence, uniqueness and continuous dependence results for an abstract class of nonlinear parabolic systems which includes the TCE PBPK-hybrid model as a special case. Moreover, we addressed theoretical issues for the associated general parameter estimation problem and established the convergence of the Galerkin finite element approximations.

\section{Acknowledgments}

This research has been supported in part by the Air Force Office of Scientific Research under grants AFOSR F49620-98-1-0180 and AFOSR F49620-01-1-0026, as well by an NSF-GRT fellowship (grant GER-9454175) and a P.E.O. Scholar Award to L.K.P.

\section{References}

[1] A. Ackleh, H. T. Banks, and G. Pintér, Well-posedness results for models of elastomers, Tech. Rep. CRSC-TR00-21, Center for Research in Scientific Computation, North Carolina State University, September 2000; Journal of Mathematical Analysis and Applications (to appear).

[2] Richard A. Albanese, H. T. Banks, Marina V. Evans, and Laura K. Potter, Physiologically based pharmacokinetic models for the transport of trichloroethylene in adipose tissue, Tech. Rep. CRSC-TR01-03, Center for Research in Scientific Computation, North Carolina State University, January 2001; Bulletin of Mathematical Biology submitted.

[3] H. T. Banks, D. S. Gilliam, and V. I. Shubov, Global solvability for damped abstract nonlinear hyperbolic systems, Differential and Integral Equations 10 (1997), 309-332.

[4] H. T. Banks and K. Kunisch, Estimation techniques for distributed parameter systems, Birkhauser, Boston, 1989.

[5] H. T. Banks and C. J. Musante, Well-posedness for a class of abstract nonlinear parabolic systems with time delay, Nonlinear Analysis - Theory, Methods \& Applications 35 (1999), 629-648. 
[6] H. T. Banks, C. J. Musante, and J. K. Raye, Approximation methods for inverse problems governed by nonlinear parabolic systems, Tech. Rep. CRSCTR99-38, Center for Research in Scientific Computation, North Carolina State University, December 1999; Numerical Functional Analysis and Optimization 21 (2000), 791-816.

[7] H. T. Banks, R. C. Smith, and Y. Wang, Smart material structures: Modeling, estimation and control, John Wiley \& Sons, Chichester, 1996.

[8] H. A. Barton and H. J. Clewell III, Evaluating noncancer effects of trichloroethylene: Dosimetry, mode of action, and risk assessment, Environmental Health Perspectives 108 Suppl. 2 (2000), 323-334.

[9] H. Brauch, G. Weirich, M. A. Hornauer, S. Störkel, T. Wöhl, and T. Brüning, Trichloroethylene exposure and specific somatic mutations in patients with renal cell carcinoma, Journal of the National Cancer Institute 91 (1999), 854-861.

[10] J. V. Bruckner, B. D. Davis, and J. N. Blancato, Metabolism, toxicity, and carcinogenicity of trichloroethylene, Critical Reviews in Toxicology 20 (1989), $31-50$.

[11] R. J. Bull, Mode of action of liver tumor induction by trichloroethylene and its metabolites, trichloroacetate and dichloroacetate, Environmental Health Perspectives 108 Suppl. 2 (2000), 241-260.

[12] Michele L. Bush, Clay B. Frederick, Julia S. Kimbell, and James S. Ultman, $A$ CFD-PBPK hybrid model for simulating gas and vapor uptake in the rat nose, Toxicology and Applied Pharmacology 150 (1998), 133-145.

[13] D. Crandall and M. DiGirolamo, Hemodynamic and metabolic correlates in adipose tissue: Pathophysiologic considerations, FASEB 4 (1990), 141-147.

[14] D. Crandall, G. J. Hausman, and J. G. Kral, A review of the microcirculation of adipose tissue: anatomic, metabolic, and angiogenic perspectives, Microcirculation 4 (1997), 211-232.

[15] M. V. Evans, W. K. Boyes, P. J. Bushnell, J. H. Raymer, and J. E. Simmons, A physiologically based pharmacokinetic model for trichloroethylene (TCE) in Long-Evans rats, Personal communication, 1999.

[16] A. R. Goeptar, J. N. M. Commandeur, B. van Ommen, P. J. van Bladeren, and N. P. E. Vermeulen, Metabolism and kinetics of trichloroethylene in relation to toxicity and carcinogenicity. relevance of the mercapturic acid pathway, Chemical Research in Toxicology 8 (1995), 3-21. 
[17] T. Green, Pulmonary toxicity and carcinogenicity of trichloroethylene: Species differences and modes of action, Environmental Health Perspectives 108 Suppl. 2 (2000), 261-264.

[18] G. J. Hausman, The comparative anatomy of adipose tissue, New Perspectives in Adipose Tissue: Structure, Function and Development, Butterworths, London, 1985.

[19] J. L. Lions and E. Magenes, Non-homogeneous boundary value problems and applications, vol. I, Springer-Verlag, New York, 1972.

[20] M. A. Medinsky and C. D. Klaassen, Toxicokinetics, Casarett and Doull's Toxicology: The Basic Science of Poisons, McGraw-Hill, Health Professions Division, New York, 5th ed., 1996.

[21] G. M. Pastino, W. Y. Yap, and M. Carroquino, Human variability and susceptibility to trichloroethylene, Environmental Health Perspectives 108 Suppl. 2 (2000), 201-215.

[22] Laura K. Potter, Physiologically based pharmacokinetic models for the systemic transport of trichloroethylene, Ph.D. thesis, North Carolina State University, Raleigh, NC, August 2001.

[23] B. D. Reddy, Introductory functional analysis: With applications to boundaryvalue problems \& finite elements, Springer-Verlag, New York, 1997.

[24] M. S. Roberts and M. Rowland, A dispersion model of hepatic elimination: 1. Formulation of the model and bolus considerations, Journal of Pharmacokinetics and Biopharmaceutics 14 (1986), 227-260.

[25] S. Rosell and E. Belfrage, Blood circulation in adipose tissue, Physiological Reviews 59 (1979), 1078-1104.

[26] A. Roy, C. P. Weisel, P. J. Lioy, and P. G. Georgopoulos, A distributed parameter physiologically-based pharmacokinetic model for dermal and inhalation exposure to volatile organic compounds, Risk Analysis 16 (1996), 147-60.

[27] A. M. Saillenfait, I. Langonne, and J. P. Sabate, Developmental toxicity of trichloroethylene, tetrachloroethylene and four of their metabolites in rat whole embryo culture, Archives of Toxicology 70 (1995), 71-82.

[28] C. S. Scott and V. J. Cogliano, Trichloroethylene health risks-state of the science, Environmental Health Perspectives 108 Suppl. 2 (2000), 159-160. 
[29] United Nations Environment Programme, International Labour Organisation, and World Health Organization, Trichloroethylene, World Health Organization, Geneva, 1985.

[30] J. Wloka, Partial differential equations, Cambridge University Press, Cambridge, 1987.

Email address: htbanks@eos.ncsu.edu 